\title{
Avaliação da repercussão dos sintomas depressivos na qualidade de vida de pacientes com DPOC
}

\author{
Evaluation of the repercussion of depressive symptoms on the quality of life of \\ patients with COPD
}

\author{
Ana Cristina Guimarães Mendes Alves ${ }^{1}$, Ana Paula Vieira Machado Ramos ${ }^{1}$, Bárbara \\ Oliveira Paixão ${ }^{1}$, Jéssica Faria Freitas ${ }^{1}$, Júnia Rios Garib ${ }^{2}$, Natália Costa Freitas ${ }^{1}$
}

\begin{abstract}
Alves ACGM, Ramos APVM, Paixão BO, Freitas JF, Garib JR, Freitas NC. Avaliação da repercussão dos sintomas depressivos na qualidade de vida de pacientes com DPOC / Evaluation of the repercussion of depressive symptoms on the quality of life of patients with COPD. Rev Med (São Paulo). 2019 nov.-dez.;98(6):374-81.
\end{abstract}

RESUMO: A doença pulmonar obstrutiva crônica (DPOC) associa-se frequentemente a múltiplas comorbidades, sendo a depressão frequente e na maioria das vezes, subdiagnosticada. Sua prevalência em pacientes portadores de DPOC varia de 10 a $42 \%$ - uma proporção muito maior que na população geral - e está relacionada a maiores taxas de exacerbações e admissões hospitalares, a maior gravidade dos sintomas, a piora da funcionalidade e por fim, ao aumento da mortalidade. Nesse contexto, a detecção do quadro depressivo em pacientes portadores de doença pulmonar obstrutiva crônica contribui para melhor qualidade de vida desses pacientes. Com o objetivo de avaliar a prevalência de sintomas depressivos em pacientes portadores de DPOC e analisar a repercussão desses sintomas na qualidade de vida, nas taxas de exacerbações, nas admissões hospitalares e na gravidade dos sintomas dos pacientes portadores dessa doença foi realizado um estudo não intervencional, descritivo e transversal no período de março de 2018 a outubro de 2018 no ambulatório de pneumologia da Faculdade Ciências Médicas de Minas Gerais. Entre os resultados, a prevalência de sintomas depressivos foi de $25 \%$. Ao contrário do que se esperava os sintomas foram mais comuns na população idosa e não houve associação significativa entre sexo e índice da função pulmonar. Os indivíduos com DPOC e sintomas depressivos apresentaram piores desfechos relacionados à qualidade de vida, exacerbações e internações hospitalares. Não foi possível comprovar se pacientes com piores padrões respiratórios desenvolvem sintomas depressivos ou se portadores de sintomas depressivos desenvolvem mais exacerbações por possivelmente apresentarem sintomatologia mais grave. Sendo necessário novos estudos para elucidar essas hipóteses.

Descritores: Transtorno depressivo; Doença pulmonar obstrutiva crônica; Qualidade de vida; Exacerbação dos sintomas; Pacientes.
ABSTRACT: Chronic obstructive pulmonary disease (COPD) is often associated with multiple comorbidities, and depression is frequent and most often underdiagnosed. The prevalence of depression in COPD patients varies from 10 to $42 \%$, a much higher proportion than in the general population, and its related to higher rates of exacerbations and hospital admissions, greater severity of symptoms, worsening of functionality and ultimately, increased mortality. In this context the detection of the depressive condition in patients with chronic obstructive pulmonary disease contributes to a better quality of life of this patients. In order to evaluate the prevalence of depressive symptoms in patients with COPD and to analyze the repercussion of these symptoms in the quality of life, in rates of exacerbations, in hospital admission and in the severity of symptoms of patients with this disease, a non-interventional, descriptive and transversal study in the period from March 2018 to October 2018 at the Pulmonology Outpatient Clinic of the Faculdade Ciências Médicas of Minas Gerais. Among the results, the prevalence of depressive symptoms was $25 \%$. Contrary of what was expected, the symptoms were more common in the elderly population and there was no significant association between sex and pulmonary function index. Individuals with COPD and depressive symptoms presented worse outcomes related to quality of life, exacerbations and hospital admissions. It was not possible to verify whether patients with worse respiratory patterns develop depressive symptoms or if patients with depressive symptoms develop more exacerbations by possibly presenting worse severe symptomatology. Further studies are needed to elucidate these hypotheses.

Keywords: Depressive disorder; Pulmonary disease, chronic obstructive; Quality of life; Symptom flare up; Patients.

1. Acadêmica de Medicina da Faculdade Ciências Médicas de Minas Gerais, Belo Horizonte - MG. ORCID: Alves ACGM - https://orcid.org/0000-00029970-1848; Ramos APVM - https://orcid.org/0000-0001-7872-1623; Paixão BO - https://orcid.org/0000-0002-7022-8989; Freitas JF - https://orcid. org/0000-0003-4378-8080; Freitas NC - https://orcid.org/0000-0002-6226-0153. Email: ana_cristina249@hotmail.com, anapaulamachadoramos@ hotmail.com, barbara_paixao@hotmail.com,jeffreitas@hotmail.com, nataliacfreitas19@gmail.com.

2. Docente do Curso de Medicina na Faculdade Ciências Médicas de Minas Gerais, Belo Horizonte - MG. ORCID: https://orcid.org/0000-0002-43391182. Email: juniagarib@hotmail.com.

Endereço para correspondência: Bárbara Oliveira Paixão/ Rua Garret, 925, aptº 202, bloco A, Jardim América, Belo Horizonte, MG. Email: barbara paixao@hotmail.com 


\section{INTRODUÇÃO}

A Doença Pulmonar Obstrutiva Crônica (DPOC) é caracterizada por limitação progressiva e irreversível ao fluxo de $\operatorname{ar}^{1}$. Apresenta alto índice de mortalidade, sendo a líder entre as doenças respiratórias. Atualmente, no Brasil ocupa o quinto lugar entre as principais causas de morte e corresponde a $25 \%$ das hospitalizações por problemas respiratórios ${ }^{1}$.

Seu curso crônico associa-se frequentemente a múltiplas comorbidades como doenças cardiovasculares, diabetes mellitus e depressão, sendo a última frequente, porém na maioria das vezes, subdiagnosticada ${ }^{2,3}$. A prevalência de depressão em pacientes com DPOC varia de 10 a 42\%, uma proporção muito maior que na população geral $^{4}$. Estudos de coorte sugerem que essa prevalência seja maior nas mulheres e nos indivíduos com maior sintomatologia respiratória, independente da classificação da limitação pulmonar (GOLD I, II, III ou IV) $)^{4,5}$.

Estudos afirmam também que a depressão está mais frequentemente associada à DPOC do que à outras doenças crônicas e doenças respiratórias ${ }^{4,-7}$. De acordo com um amplo estudo de coorte, baseado no Banco de Dados de Pesquisa em Prática Geral, a taxa de incidência de depressão foi de 16,2 por 1.000 pessoas/ano em pacientes portadores de DPOC e 9,4 por 1.000 pessoas/ano em pacientes não portadores de DPOC - o que levou a uma taxa bruta de incidência de 1,72 (intervalo de confiança de 95\%, 1,58-1,88). ${ }^{[8]}$ Além disso, uma metanálise de 2011 constatou que $24,6 \%$ dos pacientes com DPOC apresentou sintomas depressivos (Intervalo de confiança de 95\% 20,028,6 ) enquanto apenas $11,7 \%$ do grupo controle apresentou tais sintomas (Intervalo de confiança 95\% 9,0-15,1) 9-11.

Apesar dos impactos negativos da depressão sobre a doença pulmonar obstrutiva crônica serem reconhecidos em diversos estudos, seus aspectos fisiopatológicos ainda são incertos. ${ }^{[2] ~[4] ~[5] ~[7] ~ A c r e d i t a-s e ~ e m ~ u m a ~ p r e d i s p o s i c ̧ a ̃ o ~}$ genética associada a fatores ambientais como a própria doença respiratória crônica e seu efeito neuropsiquiátrico direto $^{12}$.

Indivíduos com DPOC e depressão comórbida apresentam piores desfechos.A associação das duas doenças se relaciona com maiores taxas de exacerbações, sendo que estas por si só aceleram declínio da função respiratória e aumentam o risco cardiovascular e de hospitalizações. Além das exacerbações, há elevação das admissões hospitalares, maior gravidade dos sintomas, piora da funcionalidade diária e por fim, aumento da mortalidade $2,4,5,7,13,14$. Ademais, apenas um terço dos pacientes com essa comorbidade recebe tratamento adequado ${ }^{15,16,17}$.

Portanto, a presença de uma patologia que influencie na evolução desfavorável da doença pulmonar obstrutiva crônica é uma condição que deve ser avaliada e controlada ${ }^{4,5,18}$. As diretrizes baseadas em evidências para DPOC não fornecem um esboço detalhado para uma boa abordagem, triagem e diagnóstico da depressão. Ainda assim, instrumentos de triagem práticos, como a Escala Hospitalar de Ansiedade e Depressão (HADS) e a avaliação de transtornos mentais na atenção primária, foram validados em pacientes portadores de DPOC e apresentam bom valor preditivo positivo ${ }^{4}$.

A depressão tem sido extensivamente estudada e é conhecida por ter um impacto negativo nos desfechos de pacientes portadores de DPOC. Portanto, a detecção do quadro depressivo em pacientes com doença pulmonar obstrutiva crônica é de extrema importância e contribui para melhor qualidade de vida desses pacientes ${ }^{4,5,14,18}$.

\section{CASUÍSTICA E MÉTODOS}

Trata-se de um estudo não intervencional, descritivo e transversal. $\mathrm{O}$ estudo foi conduzido no ambulatório de pneumologia da Faculdade Ciências Médicas de Minas Gerais (FCMMG) do período de março de 2018 a outubro de 2018.

Foram avaliados 48 pacientes. Os critérios de elegibilidade foram: diagnóstico estabelecido de DPOC (espirometria com Índice de Tiffeneau menor que 0,7 ou menor que o limite inferior); idade maior que 40 anos e indivíduos clinicamente estáveis. Foram excluídos da pesquisa pacientes portadores de outras doenças pulmonares como asma, bronquiectasia, doenças intersticiais e tuberculose; pacientes submetidos a cirurgia pulmonar ou com história de malignidade há pelo menos 5 anos; pacientes que se recusaram a participar da pesquisa ou foram inaptos a compreender os questionários.

Os pacientes elegíveis responderam a quatro questionários, sendo eles: CAT (COPD Assessment Test), AQ20 (Airways questionnaire 20), HADS (Hospital Anxiety and Depression Scale) - questionários traduzidos e validados ${ }^{19-22}$ - e um questionário de identificação. $\mathrm{O}$ questionário de identificação teve o objetivo de identificar e obter informações clínicas adicionais do paciente como: comorbidades, medicação de uso contínuo, tabagismo (anos/maço), exacerbações e internações hospitalares por dispneia no último ano. O critério utilizado para definição de exacerbação é descrito pelo GOLD (Global Initiative for Obstructive Lung Disease) como um evento, de início agudo, caracterizado por uma mudança na dispneia basal do paciente, tosse e/ou expectoração que estão além dos padrões normais do dia a dia.

De acordo com o estudo prospectivo multicêntrico, o CAT (COPD Assessment Test) é um instrumento que apresenta boa correlação com o questionário St. George's Respiratory Questionnaire (SGRQ) ${ }^{5}$. Em 2011, o GOLD recomendou o uso do CAT para avaliação de pacientes com DPOC $^{5}$. Por ser uma ferramenta válida e simples foi aplicada aos participantes do estudo. O teste avalia a repercussão da DPOC na funcionalidade dos pacientes por meio de oito itens: tosse, secreção, dispneia ao subir ladeira ou escada, 
aperto no peito, limitação da atividade em casa, confiança em sair de casa, sono e energia. Cada item é pontuado de 0 a 5 , sendo 5 o pior nível de sintoma e a pontuação total 40 pontos. Já o AQ20 (Airways Questionnaire 20) avalia a repercussão da DPOC na qualidade de vida diária dos pacientes por meio de 20 itens.

Com a finalidade de acessar a presença de sintomas depressivos, foi usado o HADS (Hospital Anxiety and Depression Scale). Há várias ferramentas que podem ser usadas no rastreio de pacientes com sintomas depressivos, mas poucas foram especificamente validadas para os portadores de DPOC ou outras doenças pulmonares. ${ }^{[3]}$ Uma revisão sistemática descreveu e avaliou os quatro instrumentos úteis para o rastreamento de um possível quadro depressivo, sendo eles: HADS, Beck Depression Inventory, Geriatric depression scale e Centre for Epidemiological Studies Scale on Depression. Entre eles, somente a HADS foi especificamente validada para a $\mathrm{DPOC}^{18}$. O instrumento é composto por 14 perguntas, das quais sete avaliam ansiedade (HADS-A) e sete avaliam depressão (HADS-D). Cada pergunta analisa os sintomas na última semana e pontua de zero a três. Um escore $\geq 9$ nos itens HADS-A e HADS-D é considerado como sintoma ansioso e depressivo, respectivamente. O HADS é validado e amplamente usado em estudos incluindo pacientes com DPOC.

A classificação da DPOC foi realizada de acordo com a gravidade do fluxo aéreo, sintomas respiratórios e número de exacerbações:

- GOLD I, II, III, IV;

- Grupos A, B, C e D.

A classificação da gravidade da limitação ao fluxo aéreo é determinada através do exame de espirometria. A variável que confirma o grau de severidade funcional é o volume expiratório forçado no primeiro segundo (VEF1\%). Tal parâmetro deve ser analisado após a realização da prova broncodilatadora (Pós BD) ${ }^{23}$.

- GOLD I: VEF1 $\geq 80 \%$ do valor previsto;

- GOLD II: VEF1 79-50\% do valor previsto;

- GOLD III: VEF1 49-30\% do valor previsto;

- GOLD IV: VEF $1<30 \%$ do valor previsto.

A classificação em grupos A, B, C e D baseia-se na sintomatologia respiratória e no número de exacerbações e internações hospitalares ${ }^{23}$.

- Grupo A: CAT < 10 em pacientes com 0 ou 1 exacerbação sem necessidade de atendimento de urgência;

- Grupo B: CAT $\geq 10$ em pacientes com 0 ou 1 exacerbação sem necessidade de atendimento de urgência;

- Grupo C: CAT< 10 em pacientes com 2 ou mais exacerbações sem necessidade de atendimento de urgência ou com 1 ou mais atendimentos de urgência;

- Grupo D: CAT $\geq 10$ em pacientes com 2 ou mais exacerbações sem necessidade de atendimento de urgência ou com 1 ou mais atendimentos na urgência.

As variáveis categóricas são apresentadas como frequências absolutas e relativas e as numéricas como média \pm desvio-padrão. As variáveis numéricas foram submetidas ao teste de normalidade de Shapiro-Wilk. Para comparar médias, foi usado o teste de Mann-Whitney. Para comparar a variável sexo, foi utilizado o teste QuiQuadrado. A fim de avaliar a utilidade preditiva do CAT para sintomas depressivos em pacientes portadores de DPOC, foi utilizado o Teste Exato de Fisher (avaliação da associação entre as escalas HADS e CAT).

A frequência da depressão foi determinada de acordo com sexo, idade, exacerbação e/ou internação hospitalar por dispneia no último ano, escore do AQ20 e do CAT.

As análises foram realizadas no programa $\mathrm{R}$ versão 3.4.3 e foi adotado nível de significância de 5\%.

\section{RESULTADOS}

Do total de 48 pacientes $64,58 \%$ foram do sexo feminino, com idade média de 70,75 $\pm 9,88$. O valor médio do VEF1\% pós BD foi $57,14 \pm 20,79$. A maioria dos pacientes apresentou obstrução moderada ao fluxo aéreo - GOLD II - (38,71\%) e foi classificada clinicamente como DPOC grupo B (42,22\%). As características clínicas e demográficas da amostra estão ilustradas na Tabela 1.

Tabela 1 - Característica clínica e demográfica da amostra

\begin{tabular}{|l|c|}
\hline Variáveis & $\mathbf{n}(\mathbf{\%})$ \\
\hline Idade & $70,75 \pm 9,88$ \\
\hline Sexo & $31(64,58)$ \\
\hline Feminino & $17(35,42)$ \\
\hline Masculino & $57,14 \pm 20,79$ \\
\hline VEF1 Pós BD \% & \\
\hline GOLD - Espirometria & $4(12,90)$ \\
\hline I - Leve & $12(38,71)$ \\
\hline II - Moderado & $11(35,48)$ \\
\hline III - Grave & $4(12,90)$ \\
\hline IV - Muito grave & $7(15,56)$ \\
\hline GOLD - Clínico & $19(42,22)$ \\
\hline A & $3(6,67)$ \\
\hline B & $16(35,56)$ \\
\hline C & \\
\hline D & \\
\hline
\end{tabular}

Nota: Os dados são apresentados como média \pm desvio padrão ou número absoluto (porcentagem). Definição das abreviações: VEF1 Pós \%, volume expiratório forçado no primeiro segundo pós prova broncodilatadora; GOLD, iniciativa global para doença pulmonar obstrutiva crônica. Fonte: Elaborada pelas autoras.

A prevalência de sintomas depressivos na população estudada foi de $25 \%$. Estes sintomas foram mais comuns na população idosa, principalmente na faixa etária de 6069 anos; representando $50 \%$ dos pacientes depressivos (Tabela 2). 
Alves ACGM, et al. Avaliação da repercussão dos sintomas depressivos na qualidade de vida de pacientes.

Tabela 2 - Variável idade estratificada pela presença de depressão (definida como pontuação no HADS-D maior ou igual a 9)

\begin{tabular}{|c|c|c|}
\hline & \multicolumn{2}{|c|}{ HADS - Depressão } \\
\hline & $<\mathbf{9}$ & $\geq \mathbf{9}$ \\
\hline Idade & & \\
\hline $50-59$ anos & $3(8,33)$ & $2(16,66)$ \\
\hline $60-69$ anos & $12(33,33)$ & $6(50,00)$ \\
\hline $70-79$ anos & $11(30,55)$ & $3(25,00)$ \\
\hline $80-89$ anos & $10(27,77)$ & 0 \\
\hline $90-99$ anos & 0 & $1(8,33)$ \\
\hline
\end{tabular}

Nota: Os dados são apresentados número absoluto (porcentagem). Fonte: Dados da pesquisa

A Tabela 3 lista as características basais dos indivíduos com DPOC e depressão. Os indivíduos com DPOC e sintomas depressivos apresentaram piores desfechos, relacionados à qualidade de vida, avaliados pelo CAT e pelo AQ20. No CAT 33\% dos pacientes apresentaram impacto muito grave na funcionalidade e no AQ20 os participantes apresentaram um escore mais alto, em relação a população não depressiva, sendo o valor médio $12,67 \pm 3,58$ pontos. Além disso, esses pacientes apresentaram um maior número de exacerbação $(1,83 \pm$ $1,47)$ e hospitalização no ano anterior $(1,58 \pm 1,56)$ se comparados aos pacientes com DPOC sem depressão.

Ao analisar o valor- $p$ através dos testes Quiquadrado, Fisher e Mann-Whitney foi possível determinar as relações significativas, sendo a variável "sexo" analisada pelo teste Qui-quadrado; as variáveis "exacerbação", "atendimento de urgência" e "AQ20" analisadas pelo teste de Mann-Whitney e a variável "CAT" analisada pelo teste de Fisher - os respectivos valores dos testes estão expostos na Tabela 3. Portanto, houve diferença significativa entre os sintomas depressivos, representados por pontuação maior ou igual a 9 no HADS, e o número de exacerbações, os atendimentos de urgência e as pontuações no AQ20 e no CAT.

Não houve associação significativa, entre sexo, severidade da função pulmonar (GOLD I, II, III e IV) e o resultado do HADS para depressão (Tabela 3).

Tabela 3 - Características de base de doenças pulmonares obstrutivas crônicas estratificadas pela presença de depressão (definida como pontuação no HADS-D maior ou igual a 9)

\begin{tabular}{|c|c|c|c|}
\hline & \multicolumn{2}{|c|}{ HADS - Depressão } & \multirow{2}{*}{ Valor-p } \\
\hline & $<9$ & $\geq 9$ & \\
\hline \multicolumn{4}{|l|}{ Sexo } \\
\hline Feminino & $21(58,33)$ & $10(83,33)$ & \multirow{2}{*}{$0,223^{\mathrm{Q}}$} \\
\hline Masculino & $15(41,6)$ & $2(16,67)$ & \\
\hline Exacerbações & $0,61 \pm 1,10$ & $1,83 \pm 1,47$ & $0,002^{\mathrm{M}}$ \\
\hline Atendimentos de urgência & $0,53 \pm 1,00$ & $1,58 \pm 1,56$ & $0,006^{\mathrm{M}}$ \\
\hline \multicolumn{4}{|l|}{ GOLD - Espirometria } \\
\hline I & $3(12,50)$ & $1(14,29)$ & \multirow{4}{*}{$0,926^{\mathrm{F}}$} \\
\hline II & $10(41,67)$ & $2(28,57)$ & \\
\hline III & $8(33,33)$ & $3(42,86)$ & \\
\hline IV & $3(12,50)$ & $1(14,29)$ & \\
\hline AQ20 & $7,53 \pm 4,67$ & $12,67 \pm 3,58$ & $0,002^{\mathrm{M}}$ \\
\hline \multicolumn{4}{|l|}{ CAT } \\
\hline Nenhum impacto & $3(8,33)$ & $0(0,00)$ & \multirow{5}{*}{$0,013^{\mathrm{F}}$} \\
\hline Impacto leve & $8(22,22)$ & $1(8,33)$ & \\
\hline Impacto moderado & $19(52,78)$ & $3(25,00)$ & \\
\hline Impacto grave & $5(13,89)$ & $4(33,33)$ & \\
\hline Impacto muito grave & $1(2,78)$ & $4(33,33)$ & \\
\hline
\end{tabular}

Nota: Os dados são apresentados como média \pm desvio padrão ou número absoluto (percentagem). Definição das abreviações: GOLD, iniciativa global para doença pulmonar obstrutiva crônica; AQ20, questionário de vias aéreas; CAT, teste de avaliação de DPOC; ${ }^{\mathrm{Q}}$ Teste Qui-quadrado; ${ }^{\mathrm{F}}$ Teste Exato de Fisher; ${ }^{\mathrm{M}}$ Teste de Mann-Whitney. Fonte: Dados da pesquisa.

Segundo o Teste de Fisher, a pontuação do CAT associou-se significativamente com os sintomas depressivos, de modo que quem obteve pontuações maiores ou iguais a 9 no HADS, apresentou impactos mais graves no CAT. Dessa forma, esse teste apresentou boa utilidade preditiva de depressão em pacientes com DPOC (Gráfico 1). 
Gráfico 1 - Pontuação do CAT em pacientes com sintomas depressivos

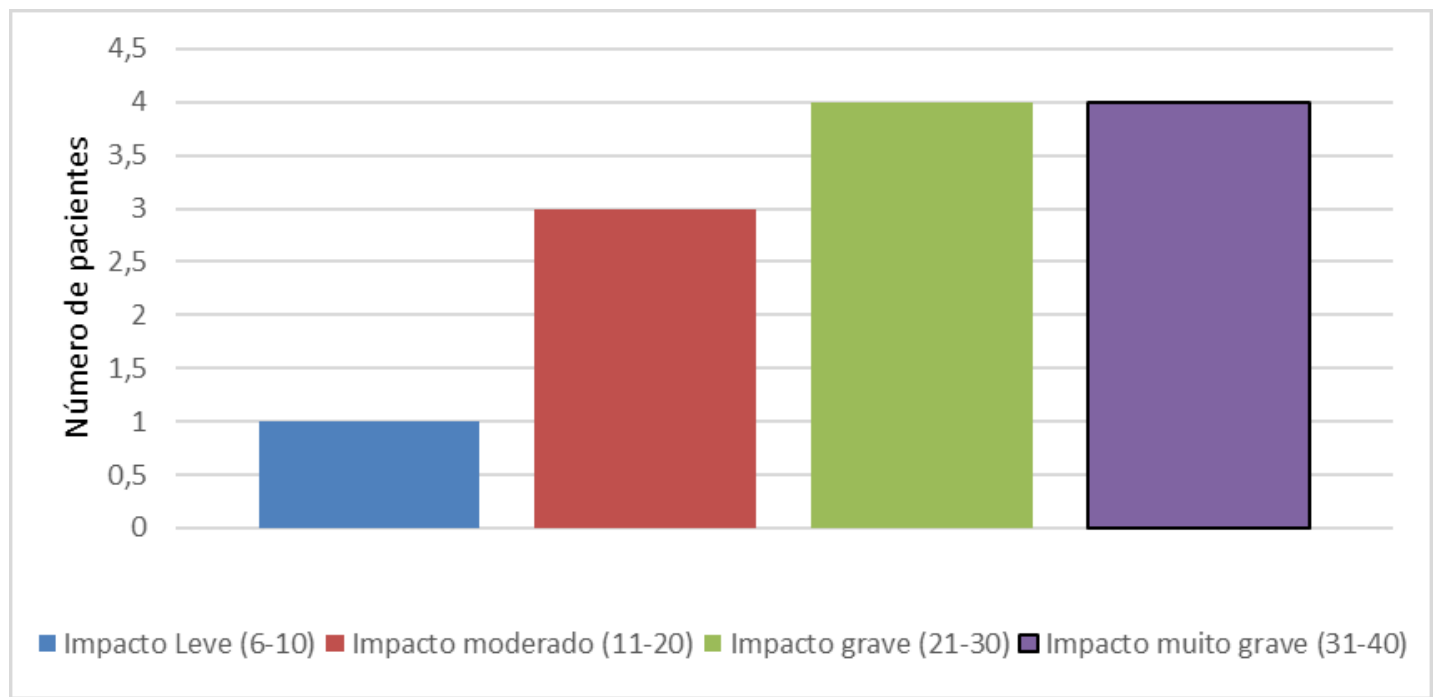

Nota: Pacientes com sintomas depressivos estratificados de acordo com impacto no questionário CAT, sendo a pontuação de 6-10 impacto leve; 11-20 impacto moderado; 21-30 impacto grave; 31-40 impacto muito grave.

Fonte: Elaborada pelas autoras.

$\mathrm{Na}$ análise individual do CAT (Tabela 4), o item "falta de ar ao subir uma ladeira ou um andar de escadas" foi o mais prevalente seguido de "limitação nas atividades em casa", correspondentes às questões 4 e 5 . Já o item "energia" presente na questão 8 , considerado o de maior poder de discriminação e o de melhor correlação com a presença de depressão no questionário CAT, não se mostrou prevalente no atual estudo.

Portanto, os dois itens mais prevalentes nesse estudo corroboram com a correlação encontrada entre sintomas depressivos e limitação física importante decorrente da sintomatologia grave da DPOC.

Tabela 4 - Associação entre os itens individuais do CAT e os sintomas depressivos

\begin{tabular}{|l|l|}
\hline CAT & Média \pm desvio-padrão \\
\hline Questão 1 & $2,31 \pm 1,59$ \\
\hline Questão 2 & $2,12 \pm 1,84$ \\
\hline Questão 3 & $1,27 \pm 1,76$ \\
\hline Questão 4 & $3,90 \pm 1,63$ \\
\hline Questão 5 & $2,83 \pm 2,19$ \\
\hline Questão 6 & $1,38 \pm 1,92$ \\
\hline Questão 7 & $1,73 \pm 1,90$ \\
\hline Questão 8 & $1,56 \pm 1,91$ \\
\hline
\end{tabular}

Nota: Questão 1 tosse; Questão 2 secreção no peito; Questão 3 pressão no peito; Questão 4 falta de ar ao subir uma ladeira ou um andar de escadas; Questão 5 limitação nas atividades em casa; Questão 6 confiança ao sair de casa apesar da doença pulmonar; Questão 7 sono; Questão 8 energia/disposição.

Fonte: Resultado da pesquisa.

\section{DISCUSSÃO}

O presente estudo apresentou achados importantes. Primeiramente, a relação entre os sintomas depressivos (avaliados pelo HADS) e a piora da funcionalidade diária (avaliada pelo CAT) foi comprovada. Essa relação pode ser explicada por meio de duas hipóteses sendo a primeira a de que os pacientes com piores padrões respiratórios desenvolvem sintomas depressivos pela própria limitação física imposta pela doença pulmonar, como descrito no estudo "Doença Pulmonar Obstrutiva Crônica - Uma Revisão". Essa limitação pode gerar no indivíduo a sensação de incapacidade e/ou inutilidade. A segunda hipótese seria o paciente já portador de sintomas depressivos, por outros fatores, que acaba desenvolvendo mais exacerbações com sintomatologia mais grave possivelmente por má adesão ao tratamento prescrito por seu médico.

Em segundo lugar, a existência de relação entre pior qualidade de vida (avaliada pelo AQ20) e presença de sintomas depressivos foi observada. A pior qualidade de vida foi relacionada à perda funcionalidade do paciente portador de DPOC. Ao se sentir incapaz de realizar certas atividades o paciente pode desenvolver sintomas depressivos.

Em terceiro lugar, pode-se observar que a classificação GOLD I, II, III e IV - de limitação da via aérea - não está tão bem correlacionada com a presença de sintomas depressivos, sendo que o escore CAT apresenta maior relação com a depressão. Isso significa que um paciente classificado como GOLD IV (VEF1<30\%) e CAT 0 (ausência de sintomatologia respiratória) apesar de sua grande limitação ao fluxo de ar, apresenta menor 
tendência a sintomas depressivos por não possuir piora da funcionalidade causada pelos sintomas graves da DPOC. Já um paciente com GOLD I (VEF1 $\geq 80 \%$ ) e CAT 40 (sintomatologia respiratória limitante) possui elevada propensão a sintomas depressivos. Esses sintomas, como dito anteriormente, podem ser decorrentes da perda da funcionalidade. Os sintomas respiratórios limitam a funcionalidade do paciente, por exemplo, por surgirem ao subir escadas, ao deambular em ambientes planos ou até mesmo podem acontecer em repouso. Assim, a frequência dos sintomas depressivos associou-se à gravidade dos sintomas e não à severidade da limitação do fluxo de ar.

Em relação às exacerbações e aos atendimentos na urgência, foi observada maior relação em pacientes com sintomas depressivos (HADS $\geq 9$ ) corroborando com os achados do estudo ECLIPSE - análise prospectiva com duração de três anos que contou com 2.118 pacientes portadores de DPOC. Não se sabe se os sintomas depressivos contribuem para a deflagração das exacerbações da doença pulmonar e dos atendimentos na urgência ou se uma má adesão à medicação gera os sintomas graves, maior frequência de atendimentos e com isso deixando o paciente mais limitado física e psicologicamente. Essas são hipóteses que poderiam ser analisadas em um outro estudo que pudesse acompanhar o paciente em um período maior de tempo para investigar mais minuciosamente a relação entre essas três variáveis.

$\mathrm{Na}$ análise da literatura, exames mais completos foram empregados como: dosagem de biomarcadores inflamatórios -entre eles proteína C-reativa, fibrinogênio, IL-8, IL-6, fator de necrose tumoral alfa - e realização de tomografia computadorizada de tórax. No entanto, esses marcadores são muito complexos para uso rotineiro em ambientes ambulatoriais. E portanto, não foram empregados no presente estudo ${ }^{4}$.

A prevalência de depressão na população estudada foi de $25 \%$, sendo mais comum em pacientes idosos do que em jovens. Não houve diferença significativa entre os sexos ou entre a gravidade da limitação das vias aéreas. Em contraste, o estudo ECLIPSE mostrou que a depressão está significativamente associada à idade mais jovem, ao sexo feminino e limitação das vias aéreas ${ }^{4}$. Em contrapartida, Lee et al. ${ }^{5}$, corroborou com o estudo atual, encontrando maior prevalência em pacientes idosos e sem diferença estatística entre o sexo ou entre a gravidade da limitação das vias aéreas. A discrepância entre o ECLIPSE e o presente estudo pode ser atribuída a diferenças amostrais - incluindo diferenças de idade, gênero e distribuição de gravidade da doença (GOLD I, II, III e IV). Pode ainda ser uma consequência das diferentes ferramentas utilizadas para avaliação e análise.

Outros autores utilizaram, previamente, vários instrumentos de rastreio para avaliar a presença de sintomas depressivos. Outras opções de questionários úteis na triagem desses sintomas são: Beck Depression Inventory, Patient Health Questionnaire-9, Geriatric depression scale e Centre for Epidemiological Studies Scale on Depression $(\mathrm{CES}-\mathrm{D})^{21}$ sendo o último utilizado no estudo ECLIPSE ${ }^{4}$. O HADS, ferramenta do presente estudo, apresenta boa confiabilidade e validade na avaliação de sintomas depressivos em pacientes portadores de DPOC. No entanto, este método, não se apresenta sem limitações, não podendo ser usado para diferenciar um diagnóstico primário de um diagnóstico secundário de depressão. Ademais, não se comporta como uma ferramenta diagnóstica e, portanto, não deve ser substituído por uma avaliação psiquiátrica abrangente ${ }^{21}$.

Quanto às limitações, primeiramente, o estudo contou com um número amostral reduzido, principalmente, devido à dificuldade e, portanto, ausência na realização da espirometria daqueles pacientes que apresentavam importante limitação da própria doença. Em segundo lugar, não foi realizada a análise da correlação dos sintomas depressivos com o tabagismo ou outras comorbidades presentes, como doenças cardiovasculares, osteoporose, diabetes mellitus, síndrome metabólica dentre outras. Possivelmente, tal análise poderia alterar os resultados encontrados no estudo, uma vez que essas doenças se correlacionam diretamente com a presença de depressão. Além disso, algumas das medicações usadas para o controle poderiam agravar os sintomas respiratórios e possivelmente os sintomas depressivos ${ }^{4}$. Por fim, dentre as várias escalas de depressão, o HADS embora seja validado para análise de depressão em pacientes com DPOC, não foi a ferramenta de escolha para os estudos usados como base para a pesquisa atual.

Apesar ser tratar de um estudo com casuística de ambulatório de um serviço específico, é possível afirmar que a população da pesquisa representa de forma acurada a população de portadores de DPOC. Os critérios de seleção da amostra, descritos previamente no item "Casuística e Método", compõem uma amostra compatível com as características sócio-demográficas da doença pulmonar obstrutiva crônica. Assim, o estudo é aplicável a outras populações.

Como ponto relevante desse estudo, grande parte das hipóteses foi corroborada. Assim, apesar das limitações, foi possível comprovar os desfechos desfavoráveis em pacientes com DPOC e depressão comórbida. O conhecimento desses desfechos é importante para os profissionais de saúde que acompanham pacientes portadores de DPOC instituírem uma abordagem terapêutica para aqueles que possuem depressão como comorbidade e instituir também um rastreio dos sintomas depressivos em todos os portadores de DPOC. Assim, a partir do momento em que se conhece as possíveis complicações dessa associação, é possível atuar para evitar evoluções desfavoráveis como exacerbações, internações e da piora funcionalidade diária. 


\section{CONCLUSÃO}

Os sintomas depressivos são conhecidos por terem um impacto negativo nos desfechos de pacientes portadores de DPOC. E apesar de sua prevalência esses sintomas são, muitas vezes, subdiagnosticados. A prevalência de sintomas depressivos na população estudada foi de $25 \%$ sem diferença significativa entre sexos, mas sendo mais prevalente em idosos.
A relação entre sintomas depressivos, gravidade dos sintomas respiratórios com consequentes exacerbações e atendimentos na urgência foi significativa. Não se sabe se os sintomas depressivos contribuem para a piora dos sintomas pulmonares ou se a sintomatologia pulmonar mais grave gera os sintomas depressivos, sendo necessário outro estudo que aborde essas hipóteses.

Contribuição dos autores: Ana Cristina Guimarães Mendes Alves - Aplicação dos questionários, revisão bibliográfica e redação do artigo; Ana Paula Vieira Machado Ramos - Aplicação dos questionários, revisão bibliográfica e redação do artigo; Bárbara Oliveira Paixão - Aplicação dos questionários, análise dos dados, revisão bibliográfica e redação do artigo; Jéssica Faria Freitas - Aplicação dos questionários, análise dos dados, revisão bibliográfica e redação do artigo; Júnia Garib Rios - Orientação e supervisão do estudo; Natália Costa Freitas - Aplicação dos questionários, revisão bibliográfica e redação do artigo.

\section{REFERÊNCIAS}

1. Laizo A. Doença pulmonar obstrutiva crónica: uma revisão. Rev Port Pneumol (Lisboa). 2009;15(6):1157-66. doi: https:// doi.org/10.1016/S0873-2159(15)30197-5.

2. Eisner MD, Anthonisen N, Coultas D, Kuenzli N, PerezPadilla R, Postma D, Romieu I, Silverman EK, Balmes JR. An official American Thoracic Society public policy statement: novel risk factors and the global burden of chronic obstructive pulmonary disease. Am J Respir Crit Care Med. 2010;182:693-718. doi: 10.1164/rccm.200811-1757ST.

3. Barnes PJ, Celli BR. Systemic manifestations and comorbidities of COPD. Eur Respir J. 2009;33(5):1165-85. doi: 10.1183/09031936.00128008.

4. Hanania NA, Müllerova H, Locantore NW, Vestbo J, Watkins ML, Wouters EMF, Rennard SI, Sharafkhaneh A. Determinants of depression in the ECLIPSE chronic obstructive pulmonary disease cohort. Am J Respir Crit Care Med. 2011;183(5):604-11. doi: 10.1164/rccm.201003-0472O.

5. Lee YS, Park S, Oh YM, Lee SD, Park SW, Kim YS, et al. Chronic obstructive pulmonary disease assessment test can predict depression: a prospective multi-center study. J Korean Med Sci. 2013;28(7):1048-54. doi: 10.3346/ jkms.2013.28.7.1048.

6. Willgoss TG, Yohannes AM. Anxiety disorders in patients with COPD: a systematic review. Respir Care. 2013;58(5):858-66. doi: $10.4187 /$ respcare. 01862 .

7. Vögele C, von Leupoldt A. Mental disorders in chronic obstructive pulmonary disease (COPD). Respir Med. 2008;102(5):764-73. doi: 10.1016/j.rmed.2007.12.006.

8. Schneider C, Jick SS, Bothner U, Meier CR. COPD and the risk of depression. Chest. 2010;137(2):341-7. doi: 10.1378/ chest.09-0614.

9. Zhang MW, Ho RC, Cheung MW, Fu E, Mak A.Prevalence of depressive symptoms in patients with chronic obstructive pulmonary disease: a systematic review, meta-analysis and meta-regression. Gen Hosp Psiquiatria. 2011;33(3):217-23. doi: 10.1016/j.genhosppsych.2011.03.009.
10. Van Ede L, Yzermans CJ, Brouwer HJ. Prevalence of depression in patients with chronic obstructive pulmonary disease: a systematic review. Tórax. 1999;54(8):688-92. doi: 10.1136/thx.54.8.688.

11. Kunik ME, K Roundy, Veazey C, Souchek J, P Richardson, Wray NP, Stanley MA. Surprisingly high prevalence of anxiety and depression in chronic breathing disorders. Chest. 2005;127(4):1205-11. doi: 10.1378/chest.127.4.1205.

12. Ng TP, Niti M, Tan WC, Cao Z, Ong KC, Eng P. Depressive symptoms and chronic obstructive pulmonary disease: effect on mortality, hospital read- mission, symptom burden, functional status, and quality of life. Arch Intern Med. 2007;167(1):60-7. doi: 10.1001/archinte.167.1.60.

13. Jordan N, Lee TA, Valenstein M, Weiss KB. Effect of care setting on evidence-based depression treatment for veterans with COPD and comorbid depression. J Gen Intern Med. 2007;22(10):1447-52. doi: 10.1007/s11606-007-0328-8.

14. Underner M, Cuvelier A, Peiffer G, Perriot J, Jaafari N. The influence of anxiety anddepression on COPD exacerbations. Rev Mal Respir. 2018;35(6):604-25. doi: 10.1016/j. rmr.2018.04.004.

15. Maurer J, Rebbapragada V, Borson S, Goldstein R, Kunik ME, Yohannes AM, Hanania NA. Anxiety and depression in COPD: current understanding, unanswered questions, and research needs. Chest. 2008; 134(4 Suppl):43S-56S. doi: 10.1378/chest.08-0342.

16. Hill K, Geist R, Goldstein RS, Lacasse Y. Anxiety and depression in end-stage COPD. Eur Respir J. 2008;31(3):66777. doi: 10.1183/09031936.00125707.

17. Júnior JLRS, Conde MB, Corrêa KS, Silva C, Prestes LS, Rabahi MF. COPD Assessment Test (CAT) score as a predictor of major depression among subjects with chronic obstructive pulmonary disease and mild hypoxemia: a case-control study. BMC Pulm Med. 2014 Nov 28;14:186. doi: 10.1186/14712466-14-186.

18. Bock K, Bendstrup E, Hilberg O, Løkke A. Screening tools for 
Alves ACGM, et al. Avaliação da repercussão dos sintomas depressivos na qualidade de vida de pacientes.

evaluation of depression in Chronic Obstructive Pulmonary Disease (COPD). A systematic review. Eur Clin Respir J. 2017;4(1):1332931. doi: 10.1080/20018525.2017.1332931.

19. Silva GP, Morano MT, Viana CM, Magalhães CB, Pereira ED. Portuguese-language version of the COPD Assessment Test: validation for use in Brazil. J Bras Pneumol. 2013;39(4):4028. doi: 10.1590/S1806- 37132013000400002.

20. Camelier A, Rosa F, Jones P, Jardim JR. Validação do questionário de vias aéreas 20 ("Airways questionnaire 20" - AQ20) em pacientes portadores de doença pulmonar obstrutiva crônica (DPOC) no Brasil. J Bras Pneumol. 2003;29(1):28-35. doi: http://dx.doi.org/10.1590/S010235862003000100007 .

21. Pais-Ribeiro J, Silva I, Ferreira T, Martins A, Meneses R, \& Baltar M. Validation study of a Portuguese version of the hospital anxiety and depression scale. Psychol Health Med. 2007;12(2):225-37. doi: 10.1080/13548500500524088.

22. Botega NJ, Bio MR, Zomignani MA, Garcia Jr C, Pereira WAB. Mood disorders among medical in-patients: a validation study of the hospital anxiety and depression scale (HAD). Rev Saude Publica. 1995;29(5);355-63. doi: http://dx.doi. org/10.1590/S0034-89101995000500004.

23. Global Initiative for Chronic Obstructive Lung Disease (GOLD). Global strategy for the diagnosis, management, and prevention of chronic obstructive lung disease 2018 Report. Available from: https://goldcopd.org/wp-content/ uploads/2017/11/GOLD-2018-v6.0-FINAL-revised-20Nov_WMS.pdf.

Recebido: 27.02.19

Aceito: 23.09.19 